IMA Journal of Applied Mathematics (2020) Page 1 of 6

doi:10.1093/imamat/xxx000

\title{
Integrable reduction and solitons of the Fokas-Lenells equation
}

\author{
THEODOROS P. HORIKIS* \\ Department of Mathematics, University of Ioannina, Ioannina 45110, Greece \\ *Corresponding author: horikis@uoi.gr \\ [Received on 10 April 2020]
}

\begin{abstract}
Novel soliton structures are constructed for the Fokas-Lenells equation. In so doing, and after discussing the stability of continuous waves, a multiple scales perturbation theory is used to reduce the equation to a Korteweg-de Vries system whose relative soliton solution gives rise to intricate (and rather unexpected) solutions to the original system. Both the focusing and defocusing equations are considered and it is found that dark solitons may exist in both cases while in the focusing case antidark solitons are also possible. These findings are quite surprising as the relative nonlinear Schrödinger equation does not exhibit these solutions.
\end{abstract}

Keywords:

Fokas-Lenells equation, $\mathrm{KdV}$ equation, dark and antidark solitons, multiple scales, modulation instability. 2020 Math Subject Classification: 35C05, 35C08, 35C20, 35Q51, 35Q55, 35 Q60.

\section{Introduction}

The theory of multiple scales analysis has been an invaluable tool in the study of physical phenomena and the underlying equations that describe them. Most of these systems, in their original form, are very difficult or even impossible to study analytically and in some cases even numerically. By the use of multiple scales theory, however, they can be reduced to more manageable systems whose properties are also quite remarkable. Prime examples are the Euler equations in water waves and Maxwell's equations in electromagnetics; the first may be reduced to the Korteweg-de Vries (KdV) and nonlinear Schrödinger (NLS) equations for shallow and deep water waves Ablowitz (2011), respectively, while the latter to the NLS equation under quasi-monochromatic approximation in optics Ablowitz (2011); Kivshar \& Agrawal (2003).

These two equations (often referred to as Universal due to the numerous applications they appear in) spawned a new field and direction in the study of nonlinear partial differential equations, namely integrable systems. These systems exhibit remarkable properties which can be systematically derived using the Inverse Scattering Transform (IST) Ablowitz \& Segur (1981) and the newly introduced extension, often referred to as the Unified Transform or Fokas method Fokas (1997).

Besides the obvious interest in integrable equations and their individual properties another important direction is the connection between them (or the reduction of one to the other). The Miura map Miura (1968) provides such a connection between the KdV and modified KdV equations, both of which are integrable. As this is not always possible, namely finding an explicit, exact way to transform one equation to another the method of multiple scales and asymptotic analysis has been employed to connect integrable systems Zakharov \& Kuznetsov (1986); Horikis \& Frantzeskakis (2014).

The purpose of this work is to provide an asymptotic connection/reduction of another NLS type integrable system to the KdV equation. We are refereing to the Fokas-Lenells (FL) equation Fokas 
(1995); Lenells \& Fokas (2009b); Lenells (2009) which is an integrable generalization of the NLS equation, derived to model nonlinear pulse propagation in monomode optical fibers when certain higherorder nonlinear effects are taken into account Lenells (2009). Mathematically, this equation is related to the NLS equation in the same way that the Camassa-Holm equation is related to the $\mathrm{KdV}$ equation Lenells \& Fokas (2009a,b) and its soliton solutions obtained using different methods Lenells \& Fokas (2009a); Lenells (2010); Vekslerchik (2011); Ai \& Xu (2019), the long time asymptotics for the system have been discussed in Ref. Xu \& Fan (2015) while its nonholonomic deformation in Ref. Kundu (2010) and rogue type solutions in Ref. Yang \& Zhang (2018); Ye et al. (2019); Geng et al. (2019). A variable coefficient system has also been studied in Refs. Lü \& Peng (2013); Wang et al. (2015, 2017) and a nonlocal variant in Ref. Zhang et al. (2019).

As such, starting from the FL system we use a multiple scales scheme that allows us to reduce the system to a KdV equation whose soliton solutions will be later used to describe solitons of the original system. Both the focusing and defocusing cases are considered. What is rather surprising is that in the focusing case (expected to be unstable as is the relative NLS limit) there is a region of stability which allows for two types of solitons to exist: dark and antidark intensity dips/humps off of a stable background. These are unique to the FL system and hence the focusing case provides a singular limit which does not fall back to the NLS case.

\section{Stability and multiplescale analysis}

To begin our analysis, consider the FL system Fokas (1997); Lenells \& Fokas (2009b); Lenells (2009)

$$
\mathrm{i} u_{t}-v u_{t x}+\gamma u_{x x}+\sigma|u|^{2}\left(u+\mathrm{i} v u_{x}\right)=0
$$

where $\gamma, v$ are real constants and $\sigma= \pm 1$. In the case of the regular NLS equation $(v=0)$, the case $\operatorname{sign}(\gamma \sigma)=1$ corresponds to the focusing case and admits bright soliton solutions (decaying to zero at infinities), while the case $\operatorname{sign}(\gamma \sigma)=-1$ is the defocusing case where dark solitons (which tend to a constant background at infinity) exist. The same terminology is used here.

Furthermore, in the focusing case the NLS equation is modulationally unstable. That means that plane/continuous waves are unstable when perturbed, exhibiting exponential growth rates. On the other hand, the defocusing case is modulationally stable. As such, the instability of plane waves that obey Eq. (2.1) is rather important and as we will see below rather interesting and somewhat different from its NLS counterpart. Indeed, consider the continuous wave (cw) solution of Eq. (2.1)

$$
u_{b}(t)=u_{0} \mathrm{e}^{\mathrm{i} u_{0}^{2} \sigma t}, \quad u_{0} \in \mathbb{R}
$$

which is perturbed as

$$
u(t, x)=\left[u_{0}+\varepsilon u_{1}(t, x)\right] \mathrm{e}^{\mathrm{i} u_{0}^{2} \sigma t}, \quad 0<\varepsilon \ll 1 .
$$

Substituting back to Eq. (2.1) and keeping terms of $O(\varepsilon)$ gives the equation for $u_{1}$,

$$
\mathrm{i} u_{1 t}-v u_{1 t x}+\gamma u_{1 x x}+u_{0}^{2} \sigma\left(u_{1}+u_{1}^{*}\right)=0
$$

where $u_{1}^{*}$ denotes the conjugate. Eq. (2.2) admits solutions of the form

$$
u_{1}(t, x)=c_{1} \mathrm{e}^{\mathrm{i}(k x-\omega t)}+c_{2} \mathrm{e}^{-\mathrm{i}(k x-\omega t)}
$$

provided the dispersion relation

$$
\left(v^{2} k^{2}-1\right) \omega^{2}+2 k v\left(\gamma k^{2}-\sigma u_{0}^{2}\right) \omega+\gamma k^{2}\left(\gamma k^{2}-2 \sigma u_{0}^{2}\right)=0 .
$$


Notice here that in the NLS limit $(v=0)$

$$
\omega^{2}=\gamma^{2} k^{4}-2 \sigma \gamma u_{0}^{2} k^{2}
$$

the sign of the product $\sigma \gamma$, used in the NLS equation, is sufficient to provide the stability conditions, namely if $\operatorname{sign}(\sigma \gamma)=1$ the (focusing) equation is unstable and when $\operatorname{sign}(\sigma \gamma)=-1$ the (defocusing) equation is stable. However, here the discriminant of Eq. (2.3) reveals that key to the stability of the equation is the product $\sigma\left(-2 \gamma+u_{0}^{2} v^{2} \sigma\right)$; when positive the equation is termed stable. Hence, the stability criterion now reads:

$$
v^{2}>2 \sigma \gamma / u_{0}^{2}, \quad \sigma^{2}=1
$$

Remarkably the same product $\sigma \gamma$ may also be used to determine stability properties, i.e. when negative the (defocusing) system is always stable. However, the focusing problem may now also be stable provided the above, leaving only a narrow window of instability. Notice also that the role of the amplitude of the $\mathrm{cw}$ should not be neglected. Indeed, the more its intensity increases the smaller the window of instability becomes. Notice that the limit $v \rightarrow 0$ is singular as the corresponding focusing NLS equation is always unstable.

The above analysis will prove to be very useful in what follows; it is the basis of the solutions which will be constructed on top of this cw. Return to Eq. (2.1) and use the Madelung transformation $u(t, x)=\rho(t, x) \exp [\mathrm{i} \phi(t, x)]$, so that after separating real and imaginary terms we get the system

$$
\begin{aligned}
& \left(1-v \phi_{x}\right) \rho_{t}+\gamma \rho \phi_{x x}+2 \gamma \rho_{x} \phi_{x}+v \sigma \rho^{2} \rho_{x}-v\left(\rho \phi_{t}\right)_{x}=0 \\
& \rho\left(1-v \phi_{x}\right) \phi_{t}+\gamma \rho \phi_{x}^{2}-\gamma \rho_{x x}-\sigma\left(1-v \phi_{x}\right) \rho^{3}+v \rho_{t x}=0 .
\end{aligned}
$$

Next define the new scales

$$
T=\varepsilon^{3 / 2} t, \quad X=\sqrt{\varepsilon}(x-c t)
$$

where $0<\varepsilon \ll 1$ a small parameter and $c$ is a travelling frame velocity to be determined later in the analysis; this is actually the speed of sound, namely the velocity of small-amplitude and long-wavelength waves propagating along the $\mathrm{cw}$ background. Furthermore, the amplitude $\rho(t, x)$ and phase $\phi(t, x)$ are expanded in a series of the small parameter as follows:

$$
\begin{aligned}
& \rho=\rho_{0}+\varepsilon \rho_{1}+\varepsilon^{2} \rho_{2}+\cdots \\
& \phi=\sigma \rho_{0}^{2} t+\sqrt{\varepsilon} \phi_{1}+\varepsilon^{3 / 2} \phi_{3} \ldots
\end{aligned}
$$

Substituting back to Eqs. (2.5)-(2.6) we obtain sets of equations defining the relative fields at different orders of $\varepsilon$. Hence at

$$
\begin{array}{rll}
O(\varepsilon): & \frac{\partial \phi_{1}}{\partial X}=-\frac{2 \sigma \rho_{0}}{c} \rho_{1} \\
O\left(\varepsilon^{3 / 2}\right): & \frac{\partial^{2} \phi_{1}}{\partial X^{2}}=\frac{c}{(\gamma+c v) \rho_{0}} \frac{\partial \rho_{1}}{\partial X}
\end{array}
$$

the compatibility condition between the two equations (the latter is obtained by differentiating the first with respect to $X$ ) yields the equation for $c$, namely:

$$
c^{2}+\left(2 \sigma v \rho_{0}^{2}\right) c+2 \gamma \sigma \rho_{0}^{2}=0 \Rightarrow c=-v \sigma \rho_{0}^{2} \pm \rho_{0} \sqrt{-2 \gamma \sigma+v^{2} \rho_{0}^{2}}
$$


Importantly one should notice here that the sign of $\left(-2 \gamma \sigma+v^{2} \rho_{0}^{2}\right)$ is also determined by the stability criterion (2.4), thus suggesting that only stable waves will propagate with real velocities and vice versa. The two signs in Eq. (2.9) correspond to waveforms propagating with different velocities. When $v=0$, i.e. in the NLS case the distinction is trivial: waveforms propagate either to the left or to the right.

Moving to the higher orders in $\varepsilon$ we obtain:

$$
\begin{aligned}
O\left(\varepsilon^{2}\right): \quad & \frac{\partial \phi_{1}}{\partial T}+(\gamma+c v)\left(\frac{\partial \phi_{1}}{\partial X}\right)^{2}+\frac{-c+2 v \rho_{0}^{2} \sigma}{\rho_{0}} \rho_{1} \frac{\partial \phi_{1}}{\partial X} \\
& -\frac{\gamma+c v}{\rho_{0}} \frac{\partial^{2} \rho_{1}}{\partial X^{2}}-3 \sigma \rho_{1}^{2}=c \frac{\partial \phi_{2}}{\partial X}+2 \rho_{0} \sigma \rho_{2} \\
O\left(\varepsilon^{5 / 2}\right): \quad & \frac{\partial \rho_{1}}{\partial T}+(\gamma+c v)\left(\rho_{1} \frac{\partial^{2} \phi_{1}}{\partial X^{2}}+2 \frac{\partial \rho_{1}}{\partial X} \frac{\partial \phi_{1}}{\partial X}\right)-v \rho_{0} \frac{\partial^{2} \phi_{1}}{\partial T \partial X} \\
& +2 v \sigma \rho_{0} \rho_{1} \frac{\partial \rho_{1}}{\partial X}=-\rho_{0}(\gamma+c v) \frac{\partial^{2} \phi_{2}}{\partial X^{2}}+c \frac{\partial \rho_{2}}{\partial X}
\end{aligned}
$$

These equations may be uncoupled if, say, one solves for $\rho_{2}$ the first, substitutes in the second and use Eq. (2.9) to eliminate $\rho_{2}$ and $\phi_{2}$. The resulting equations is:

$$
4 \rho_{0}^{2}\left(\sigma c+v \rho_{0}\right)^{2} \frac{\partial \rho_{1}}{\partial T}-2 \sigma \rho_{0}^{2}(\gamma+c v)^{2} \frac{\partial^{3} \rho_{1}}{\partial X^{3}}-12 \rho_{0}^{3}(2 \gamma+c v) \rho_{1} \frac{\partial \rho_{1}}{\partial X}=0
$$

This is clearly a $\mathrm{KdV}$ equation whose solutions and properties may now be used for the construction of solution of the original FL equation.

\section{Soliton solutions}

We are focusing here in the single soliton solution of Eq. (2.12) which may be written as

$$
\rho_{1}(T, X)=\frac{2(\gamma+c v)^{2}}{(2 \gamma+c v) \sigma \rho_{0}} \eta^{2} \operatorname{sech}^{2}\left[\eta\left(X+\frac{2(\gamma+c v)^{2}}{c+v \sigma \rho_{0}^{2}} \eta^{2} T\right)\right]
$$

with corresponding phase, obtained from Eq. (2.7),

$$
\phi_{1}(T, X)=-\frac{4(\gamma+c v)^{2}}{c(2 \gamma+c v)} \eta \tanh \left[\eta\left(X+\frac{2(\gamma+c v)^{2}}{c+v \sigma \rho_{0}^{2}} \eta^{2} T\right)\right]
$$

Of particular interest is the sign of the amplitude $\rho_{1}$, as based on the multiscale expansion the complete solution of Eq. (2.1) is written as, to $O(\varepsilon)$,

$$
u(t, x)=\left(\rho_{0}+\varepsilon \rho_{1}\right) \mathrm{e}^{\mathrm{i}\left(\sigma \rho_{0}^{2} t+\sqrt{\varepsilon} \phi_{1}\right)} .
$$

As such, depending on this sign one can have intensity dips off of the constant background $\rho_{0}$, corresponding to dark solitons or intensity humps on top of the background corresponding to antidark solitons.

In what follows we fix $\rho_{0}=1$, with no loss of generality. To fully understand the plethora of different solutions two cases will be considered: 
1. The defocusing case: Here we have $\sigma \gamma=-1$ and regardless of the values of $v$ Eq. (2.1) is always modulationally stable. Setting $\sigma=-\gamma=-1$ we can only obtain dark solitons (the sign of Eq. (3.1) is always negative) and two propagating directions. One to the left with $c_{L}=v-\sqrt{v^{2}+2}$ and one to the right with $c_{R}=v+\sqrt{v^{2}+2}$.

2. The focusing case: Remarkably the equation exhibits soliton solutions with nonzero boundary condition (provided the stability criterion $v^{2}>2 \sigma \gamma$ is respected) even in the focusing case. Moreover, two different solitons exist. Indeed, as before let us set $\sigma=\gamma=1$ then dark solitons $\left(\rho_{1}\right.$ is negative) exist propagating to the right with $c_{R}=-v-\sqrt{v^{2}-2}$ and antidark solitons ( $\rho_{1}$ is positive) exist that propagate to the left with $c_{L}=-v+\sqrt{v^{2}+2}$.

Note, finally, that in the case $\operatorname{sign}(\gamma / v)=1$ and by replacing $u(t, x)$ by $u(t,-x)$ a gauge transformation of the form $u \rightarrow \sqrt{\gamma / v^{3}} \exp (\mathrm{i} x / v) u$ transforms Eq. (2.1) into Lenells \& Fokas (2009a)

$$
u_{t x}+\frac{\gamma}{v^{3}} u-\frac{2 \mathrm{i} \gamma}{v^{2}} u_{x}-\frac{\gamma}{v} u_{x x}-\frac{\mathrm{i} \gamma}{v^{3}} \sigma|u|^{2} u_{x}=0
$$

as such the relative analysis above also refers to the solutions of this equation as well.

Some comments are important here. The IST for Eq. (2.1) with nonzero boundary conditions has been presented in Zhao \& Fan (2019) for the focusing case. In Ref. Ling et al. (2018) a plethora of single soliton are found for the coupled system. However, much like the coupled NLS equation, a coupled system may allow for more intricate soliton pairs which only exist in the coupled case, not the single equation case. For this they are often termed symbiotic solitons.

\section{Conclusions}

Two integrable equations have been asymptotically connected using a multiple scales scheme. The FL equation, derived to describe nonlinear pulse propagation in monomode optical fibers when certain higher-order nonlinear effects are taken into account is asymptotically reduced to a KdV equation, usually used in the theory of shallow water waves. As such, the single soliton solution of the latter can be used to construct (small amplitude) soliton solutions of the first. Surprisingly, the FL equation is modulationally stable even in the focusing case, where its NLS equation counterpart is always unstable. This allows for stable solutions to exist both in the defocusing and focusing regimes: only dark in the first, both dark and antidark in the latter.

It is also important to mention here that this method has also been used to describe solitons in nonlocal equations as these are formulated to describe beam propagation in nematic liquid crystals Horikis (2015). In fact, in that context many intricate solutions and relative dynamics have been revealed in coupled Horikis \& Frantzeskakis (2016) and 2D systems Horikis \& Frantzeskakis $(2017,2019)$ which encourage us to study coupled and 2D FL systems in a similar fashion. We intend to do so in a future communication.

\section{REFERENCES}

Ablowitz, M. J. (Cambridge, 2011) Nonlinear dispersive waves: Asymptotic analysis and solitons. Cambridge University Press.

Ablowitz, M. J. \& Segur, H. (1981) Solitons and the Inverse Scattering Transform. SIAM Studies in Applied Mathematics.

Ai, L. \& Xu, J. (2019) On a Riemann-Hilbert problem for the Fokas-Lenells equation. Appl. Math. Lett., 87, 57-63. Fokas, A. S. (1995) On a class of physically important integrable equations. Physica D, 87, 145-150. 
6 of 6

Fokas, A. S. (1997) A unified transform method for solving linear and certain nonlinear PDEs. Proc. Roy. Soc. Lond.A, 453, 1411-1443.

Geng, X., Shen, J. \& Xue, B. (2019) A Hermitian symmetric space Fokas-Lenells equation: Solitons, breathers, rogue waves. Ann. Phys., 404, 115-131.

Horikis, T. P. (2015) Small-amplitude defocusing nematicons. J. Phys. A: Math. Theor, 48, 02FT01.

Horikis, T. P. \& Frantzeskakis, D. J. (2014) On the NLS to KDV connection. Rom. Journ. Phys., 59, 195-203.

Horikis, T. P. \& Frantzeskakis, D. J. (2016) Vector nematicons: Coupled spatial solitons in nematic liquid crystals. Phys. Rev. A, 94, 053805.

Horikis, T. P. \& Frantzeskakis, D. J. (2017) Light Meets Water in Nonlocal Media: Surface Tension Analogue in Optics. Phys. Rev. Lett., 118, 243903.

Horikis, T. P. \& Frantzeskakis, D. J. (2019) Patterns of water in light. Proc. Royal Soc. A, 475, 20190110.

Kivshar, Y. S. \& Agrawal, G. P. (2003) Optical Solitons: From Fibers to Photonic Crystals. Academic Press.

Kundu, A. (2010) Two-fold integrable hierarchy of nonholonomic deformation of the derivative nonlinear Schrödinger and the Lenells-Fokas equation. J. Math. Phys., 51, 022901.

Lenells, J. (2009) Exactly Solvable Model for Nonlinear Pulse Propagation in Optical Fibers. Stud. Appl. Math., 123, 215-232.

Lenells, J. (2010) Dressing for a Novel Integrable Generalization of the Nonlinear Schrödinger Equation. J. Nonlinear Sci., 20, 709-722.

Lenells, J. \& Fokas, A. S. (2009a) An integrable generalization of the nonlinear Schrödinger equation on the halfline and solitons. Inverse Probl., 25, 115006.

Lenells, J. \& Fokas, A. S. (2009b) On a novel integrable generalization of the nonlinear Schrödinger equation. Nonlinearity, 22, 11-27.

Ling, L. M., Feng, B. F. \& Zhu, Z. N. (2018) General soliton solutions to a coupled Fokas-Lenells equation. Nonlinear Anal. RWA, 40, 185-214.

Lü, X. \& Peng, M. (2013) Nonautonomous motion study on accelerated and decelerated solitons for the variablecoefficient Lenells-Fokas model. Chaos, 23, 013122.

Miura, R. M. (1968) Korteweg-de Vries equation and generalizations. I. A remarkable explicit non-linear transformation. J. Math. Phys., 9, 1202-1204.

Vekslerchik, V. E. (2011) Lattice representation and dark solitons of the Fokas-Lenells equation. Nonlinearity, 24, $1165-1175$.

Wang, L., Zhu, Y.-J., Qi, F.-H., Li, M. \& Guo, R. (2015) Modulational instability, higher-order localized wave structures, and nonlinear wave interactions for a nonautonomous Lenells-Fokas equation in inhomogeneous fibers. Chaos, 25, 063111.

Wang, Z.-Q., Wang, X., Wang, L., Sun, W.-R. \& Qi, F.-H. (2017) Higher-order Peregrine combs and Peregrine walls for the variable-coefficient Lenells-Fokas equation. Superlattices Microstruct., 102, 189-201.

Xu, J. \& Fan, E. (2015) Long-time asymptotics for the Fokas-Lenells equation with decaying initial value problem: Without solitons. J. Differential Equations, 259, 1098-1148.

Yang, J. \& Zhang, Y. (2018) Higher-order rogue wave solutions of a general coupled nonlinear Fokas-Lenells system. Nonlinear Dyn., 93, 585-597.

Ye, Y., Y, Z., Chen, S., Baronio, F. \& Grelu, P. (2019) General rogue wave solutions of the coupled Fokas-Lenells equations and non-recursive Darboux transformation. Proc. R. Soc. A, 475, 20180806.

Zakharov, V. E. \& Kuznetsov, E. A. (1986) Multi-scale expansions in the theory of systems integrable by the inverse scattering transform. Physica D, 18, 455-463.

Zhang, Q., Zhang, Y. \& Ye, R. (2019) Exact solutions of nonlocal Fokas-Lenells equation. Appl. Math. Lett., 98, 336-343.

Zhao, Y. \& Fan, E. (2019) Inverse scattering transformation for the Fokas-Lenells equation with nonzero boundary. arxiv, nlin.SI, 1912.12400. 